\title{
Composición y distribución de hepáticas (Marchantiophyta) en un intervalo altitudinal en la Cordillera Oriental de Colombia
}

\author{
Cristhian Fernando Cacua-Toledo ${ }^{1}$, Víctor Hugo Serrano-Cardozo ${ }^{1}$ \& \\ Martha Patricia Ramírez-Pinilla ${ }^{1}$ \\ 1. Grupo de Estudios en Biodiversidad, Escuela de Biología, Facultad de Ciencias, Universidad Industrial de \\ Bucaramanga,Colombia; criscacua93@gmail.com,vserrano@uis.edu.co,mpramir@uis.edu.co
}

Recibido 26-IX-2017. Corregido 09-I-2018. Aceptado 05-II-2018.

\begin{abstract}
Composition and distribution of liverworts (Marchantiophyta) in a continuous altitudinal range on the Cordillera Oriental of Colombia. The species composition of liverworts varies with altitude in the Northern part of the Andes due to the relationship of biotic (vegetation) and abiotic factors (temperature, humidity, and sunlight brightness). In order to test this affirmation we determined the diversity, species composition, abundance, and distribution of species of Marchantiophyta in an altitudinal range from 2400 to $3400 \mathrm{~m}$ on the Colombian Cordillera Oriental, and established how these vary with altitude and life-zones, regarding abiotic factors. Samples, taken every $200 \mathrm{~m}$ in the altitudinal range, and environmental data were registered during a year. We found 162 species, the composition of liverworts varied throughout the altitudinal gradient with a high number of unique species in each altitude. The maximum diversity was found at $3000 \mathrm{~m}$, along with a dominance of leafy habit and epiphytic species, while the higher richness of families and genera was found between 3200 and $3400 \mathrm{~m}$. Anoplolejeunea conferta had the largest value of coverage in the zone, the highest value of importance value index (IVI), and the widest altitudinal distribution, from 2400 to $3000 \mathrm{~m}$ in four different substrates. The liverworts were distributed differently with altitude, we found a high beta diversity $(0.864)$ due to the replacement of species, with more that $30 \%$ of dissimilarity in species composition every 200 altitudinal meters, mainly depending on sunlight brightness variation. Accordingly, we found that abiotic factors like temperature, humidity, and sunlight brightness and its relationship vegetation are determinant in the diversity and altitudinal distribution of liverworts in the study area. Rev. Biol. Trop. 66(2): 559-570. Epub 2018 June 01.
\end{abstract}

Key words: ecology; bryophytes; andean forest; Marchantiophyta; altitudinal gradient.

Las hepáticas son plantas no vasculares de reducido tamaño que se encuentran distribuidas por casi todo el mundo, excepto en ambientes marinos (Gignac, 2001). Se encuentran ubicadas taxonómicamente en la división Marchantiophyta, junto con los musgos y antoceros conforman un grupo no natural conocido como briófitos, el cual no representa un nivel taxonómico (Crandall-Stotler, Stotler, \& Long, 2009).

Se llega a calcular que el número de especies de hepáticas en el mundo es cerca de los 6000, de las cuales se ubican 1350 especies en el Neotrópico distribuidas en 188 géneros (Barbosa, Uribe, \& Campos, 2007; von Konrat, Renner, Söderström, Hagborg, \& Mutke, 2008). El territorio colombiano es muy rico en especies de hepáticas, éstas se encuentran distribuidas en diferentes ambientes por todo el país; se puede encontrar casi el $60 \%$ de las especies del total de América tropical, además de poseer una sexta parte de las especies de todo el mundo (Uribe \& Gradstein, 1999). Colombia es el segundo país de América en diversidad de hepáticas con unas 713 especies (Campos, Gradstein, Uribe, \& ter Steege, 2014), después de Brasil que ocupa el primer lugar en diversidad de hepáticas con 725 especies (Gradstein \& Costa, 2003). 
La mayor riqueza de hepáticas en Colombia se encuentra concentrada en la región Andina, entre los 2000 y 3000 m (Uribe \& Gradstein, 1999), lo que equivale al $25 \%$ del territorio nacional. Esta concentración de riqueza se debe a diferentes factores como, radiación solar continua, bajas temperaturas y precipitaciones constantes (Wolf, 1993, 1994). Varios trabajos realizados en el norte de los Andes han concluido que la riqueza de hepáticas aumenta con la altitud, llegando a un pico máximo en la zona de bosque alto andino (Aguirre \& Ruiz, 2001; Barbosa et al., 2007; Churchill \& Linares, 1995; Orrego, 2005).

Para la región Andina colombiana se registran varios trabajos sobre las hepáticas en donde se compara su diversidad y distribución altitudinal (Barbosa et al., 2007; Lagos, Sáenz, \& Morales, 2008; Vargas \& Morales, 2014), distribución vertical (Gil \& Morales, 2014), distribución sobre sustratos (Pinzón \& Linares, 2006), en relación con zonas de vida (Aguirre \& Ruiz, 2001; Barbosa et al., 2007; Ruiz \& Aguirre, 2003) y de acuerdo con diferentes factores ambientales (Corrales, Duque, Uribe, \& Londoño, 2010). Sin embargo, no se han desarrollado trabajos en diversidad de hepáticas y sus aspectos ecológicos en un gradiente altitudinal continuo donde se incluyan bosque andino y páramo hacia la zona centro-norte de la cordillera Oriental.

La distribución de las hepáticas no es igual en los diferentes hábitats, depende de factores microambientales como temperatura, humedad, $\mathrm{pH}, \mathrm{y}$ radiación solar (Corrales et al., 2010; Delgado \& Ederra, 2013; Silva, Santos, \& Pôrto, 2014). También presentan una relación con la composición de la vegetación fanerógama y la disponibilidad de sustratos (Mota de Oliveira \& teer Steege, 2013; Ruiz \& Aguirre, 2003; Wolf, 1995). Debido a estos factores se asume el supuesto de una distribución diferencial de las hepáticas a través de un gradiente altitudinal continuo con preferencias de microhábitats para diferentes especies.

El objetivo del presente trabajo es determinar la riqueza composición de especies abundancia y distribución de las hepáticas presentes a lo largo de una franja altitudinal (2400 - 3400 m) en la vertiente occidental de la cordillera Oriental. Se pretende evidenciar las diferencias de composición y distribución de especies respecto de las franjas altitudinales y su relación con la temperatura, humedad y radiación solar.

\section{MATERIALES Y MÉTODOS}

Área de estudio: Se ubica en la vereda Esparta hacia el norte del municipio de Santa Bárbara departamento de Santander en el flanco occidental de la Cordillera Oriental colombiana $\left(7^{\circ} 00^{\prime} 59.4^{\prime \prime} \mathrm{N}-72^{\circ} 53\right.$ ' $51.1^{\prime \prime} \mathrm{W} \&$ $\left.7^{\circ} 02^{\prime} 26.8^{\prime} \mathrm{N} 72^{\circ}-52^{\prime} 56.6^{\prime} \mathrm{W}\right)$. El intervalo se encuentra desde los 2400 hasta los $3400 \mathrm{~m}$. Presenta un régimen de lluvias bimodal con un pico máximo en octubre y otro menor en mayo. El área cuenta con tres zonas de vida delimitadas por su altitud y vegetación (Rangel-Ch., 2000). El bosque andino (2300 a $2700 \mathrm{~m})$, bosque alto andino $(2700$ a los $3100 \mathrm{~m})$ y región paramuna (3100 $\mathrm{m}$ en adelante).

Muestreo: Se instalaron dos parcelas georreferenciadas de $100 \mathrm{~m}^{2}$ cada $200 \mathrm{~m}$ altitudinales desde los 2400 a los $3400 \mathrm{~m}$; las zonas de muestreo se seleccionaron dada la baja intervención antrópica y topografía del lugar. Se realizaron diez levantamientos por cada uno de los sustratos presentes (corteza de árbol, madera en descomposición, hojarasca, suelo desnudo, roca y raíz aflorante). La recolecta y el registro de datos se hizo según Churchill y Linares (1995), las muestras se depositaron individualmente en bolsas de papel kraft marcadas con el acrónimo del colector (CFC) y el número de colecta.

Registro de datos ecológicos y ambientales: Los datos tomados para los análisis ecológicos fueron: cobertura como dato de abundancia, a través de una plantilla de acetato de $20 \times 20 \mathrm{~cm}$ (Iwatzuki, 1960), tipo de sustrato y altitud. Adicionalmente se registraron datos ambientales durante un año a la misma altitud de las parcelas mediante miniestaciones climáticas (WatchDog Data Loggers serie 
$1000^{\circledR)}$, las cuales registraron la temperatura y humedad relativa, mientras que para el caso de humedad del suelo y luminosidad se utilizó un medidor de humedad-suelo (SP066400 ${ }^{\circledR}$ y un luxómetro lx $1330 \mathrm{~B}^{\circledR}$ ).

Análisis de datos: La determinación taxonómica de las muestras se realizó en el Laboratorio de Ecología de la Universidad Industrial de Santander utilizando estereoscopio y microscopía óptica y siguiendo claves especializadas (Gradstein, 1994; Gradstein, Churchill, \& Salazar-Allen, 2001; Uribe \& Aguirre, 1995, 1997; Teeuwen, 1989). Adicionalmente se revisó la colección de briófitas en línea del Herbario de la Universidad Nacional (COL) y se visitó el Herbario de la Universidad Pedagógica y Tecnológica de Colombia (UPTC), finalmente las muestras fueron ingresadas al Herbario de la Universidad Industrial de Santander (UIS).

Se calculó la complejidad del muestreo en cada altitud mediante el estimador de cobertura, ya que es menos sesgado de la completitud de la muestra que los estimadores no paramétricos, permitiendo comparar la diversidad con base en rarefacción/extrapolación (Chao \& Jost, 2012). Se obtuvieron los índices de diversidad en términos de números equivalentes (números de Hill) (Jost, 2006, 2010), diversidad de orden $0\left({ }^{0} \mathrm{D}\right)$ o riqueza de especies, orden $1\left({ }^{1} \mathrm{D}\right)$, que es el exponencial de la entropía del índice de Shannon, y orden 2 $\left({ }^{2} \mathrm{D}\right)$, que es el inverso del índice de Simpson. Los órdenes de estas medidas de diversidad tienen diferentes niveles de sensibilidad a la abundancia relativa de cada especie, ${ }^{0} \mathrm{D}$ considera a todas las especies con igual frecuencia, ${ }^{1} \mathrm{D}$ pesa moderadamente la abundancia de las especies y puede ser interpretado como un índice que tiene en cuenta las especies típicas $\mathrm{y}$, finalmente, ${ }^{2} \mathrm{D}$ incluye sólo aquellas especies más abundantes, la relación de cambio entre cada uno de estos índices permite construir un perfil de diversidad donde las comunidades más equitativas presentan un reducido cambio entre cada orden de diversidad (Chao et al., 2014). Se estimó la diversidad beta mediante el índice de complementariedad de Jaccard y adicionalmente se siguió el criterio de Baselga (2010) para conocer la diferenciación entre las altitudes y si esta diferencia es ocasionada por procesos de anidamiento o recambio de especies (Carvalho, Cardoso, \& Gomes, 2012; Carvalho, Cardoso, Borges, Schmera, \& Podani, 2013). Para los análisis de diversidad alfa y beta se utilizó el programa R, paquete estadístico Betapart (Baselga \& Orme, 2012) y paquete iNEXT (Hsieh, Ma, \& Chao, 2016).

La heterogeneidad ambiental en la distribución de especies se determinó mediante un análisis de correspondencia canónica (CCA), debido a que en este método de arreglo la composición de especies se relaciona directamente con las variables ambientales, conociendo si estos factores ambientales están afectando la distribución de hepáticas en el gradiente (Sánchez-González \& López-Mata, 2003; Álvarez-Yépis, Martínez-Yrízar, Búrquez, \& Lindquist, 2008; Gelviz-Gelvez \& Pavón-Hernández, 2013). Para ello se calculó el Índice de Valor de Importancia (IVI) para cada una de las especies (según Zepeda et al., 2014), calculando la densidad relativa, cobertura relativa y frecuencia. El CCA permite organizar las especies utilizando los valores de IVI y las variables ambientales tomadas. Además se realizó una prueba de ANOVA para establecer diferencias en los factores ambientales a lo largo del gradiente. Los paquetes estadísticos utilizados para los análisis fueron Past v.1.30 (Hammer, Harper, \& Ryan, 2001), Statistica 7 (Hilbe, 2007).

\section{RESULTADOS}

A lo largo del gradiente altitudinal se realizaron 720 levantamientos en las 12 parcelas seleccionadas, se encontraron 162 especies (incluyen morfoespecies) distribuidas en 46 géneros y 22 familias. Las familias más diversas fueron Lejeuneaceae (42 especies), Plagiochilaceae (31 especies), seguidas de Metzgeriaceae y Jubulaceae con 14 especies cada una. Los géneros más diversos fueron Plagiochila (31), Meztgeria (14), Lejeunea (14) y Frullania (13). Las especies con la 
CUADRO 1

Cobertura del muestreo y Diversidad de especies (en números efectivos), de las hepáticas encontradas en el gradiente altitudinal

TABLE 1

Sampling completeness and alpha diversity of the liverworts found in the altitudinal gradient

\begin{tabular}{ccccc}
\hline Altitud & Cobertura del muestreo & Riqueza de especies $\left({ }^{0} \mathrm{D}\right)$ & Diversidad de Shannon $\left({ }^{1} \mathrm{D}\right)$ & Diversidad de Simpson $\left({ }^{2} \mathrm{D}\right)$ \\
2400 & $84.6(78.9-90.4)$ & $43(36.60-49.39)$ & $28.86(23.89-33.82)$ & $18.88(12.54-25.23)$ \\
2600 & $88.9(85.7-92.0)$ & $37(31.42-42.57)$ & $26.98(22.50-31.46)$ & $21.43(17.26-25.60)$ \\
2800 & $86.9(0.82-91.7)$ & $40(34.73-45.26)$ & $25.52(21.33-29.72)$ & $17.03(12.87-21.19)$ \\
3000 & $88.0(83.4-92.7)$ & $49(44.00-53.99)$ & $39.75(34.97-44.53)$ & $33.63(28.98-38.29)$ \\
3200 & $84.1(77.6-90.7)$ & $36(29.62-42.37)$ & $25.91(20.54-31.28)$ & $20.12(14.95-25.29)$ \\
3400 & $84.1(75.1-91.1)$ & $35(29.30-40.69)$ & $24.37(18.90-29.84)$ & $16.81(11.35-22.26)$ \\
\hline
\end{tabular}

Los intervalos de confianza inferior y superior del $95 \%$ para cada valor de diversidad se indican en paréntesis.

The lower and upper $95 \%$ confidence intervals for each diversity measure are given in brackets.

mayor cobertura registrada son Anoplolejeunea conferta $\left(2512 \mathrm{~cm}^{2}\right)$ y Bazzania affinis $\left(2343 \mathrm{~cm}^{2}\right)$. El IVI muestra el peso ecológico de las especies para todo el gradiente altitudinal, A. conferta es la más importante, con un valor del IVI de 11.332 seguida de B. affinis y L. flava con 7.44 y 7.24 respetivamente. Con respecto al tipo de hábito, el folioso, fue predominante con 141 especies $(87 \%)$, mientras que el taloso registró 21 especies (13\%). El sustrato más frecuente para el establecimiento de las hepáticas fue la corteza de los árboles (epífitas) con el $58 \%$ de las especies registradas, seguido de por la madera (lignícolas) con un $55 \%$ de las especies registradas (Anexo 1).

De los seis puntos altitudinales de muestreo, en los $3000 \mathrm{~m}$ se obtuvo la mayor riqueza de especies (30\%), mientras que en los $3400 \mathrm{~m}$ se presentó la mayor riqueza de familias $(72 \%)$ y géneros $(47 \%)$ (Fig. 1). La cobertura media del muestreo fue de $86 \%$ (84-88 \%). La diversidad de Shannon (diversidad de orden $\mathrm{q}=1$ ) vario significativamente en el gradiente, presentando un valor máximo (39.75) a los $3000 \mathrm{~m}$, mientras que a los $3400 \mathrm{~m}$ presentó el menor valor de diversidad (24.37), así mismo el mayor cambio de diversidad se presentó entre los 2800 y los $3000 \mathrm{~m}$, ganando el $55.9 \%$ de la diversidad de hepáticas, mientras entre los 3000 - $3200 \mathrm{~m}$ se presentó una disminución del $34.7 \%$ de la diversidad de hepáticas (Cuadro 1). Igualmente, el mayor valor la diversidad de Simpson (diversidad orden $\mathrm{q}=2$ ) se registró a los 3000 m con 33.6 (Cuadro 1).

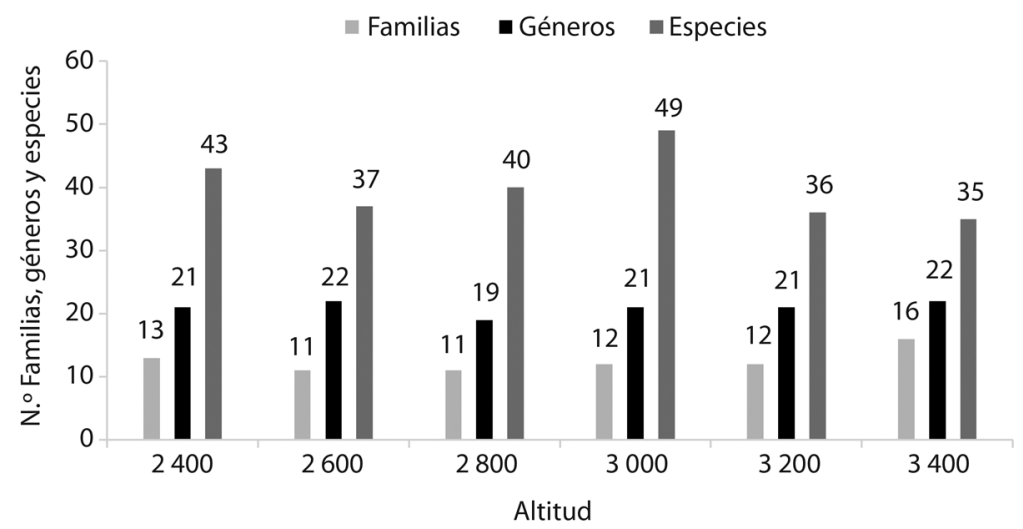

Fig. 1. Número de familias, géneros y especies de hepáticas registradas por altitud.

Fig. 1. Number of liverworts families, genera and species recorded for each of the evaluated elevations. 
CUADRO 2

Diversidad beta, valores del anidamiento (por encima de la diagonal) y del recambio (por debajo de la diagonal) de las combinaciones entre las altitudes

TABLE 2

Beta diversity, nestedness values (above the diagonal) and turnover (below the diagonal) of the combinations among elevations

\begin{tabular}{|c|c|c|c|c|c|c|c|}
\hline \multicolumn{8}{|c|}{ Anidamiento / Nestedness } \\
\hline \multirow{7}{*}{ 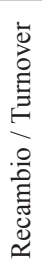 } & Altitudes & 2400 & 2600 & 2800 & 3000 & 3200 & 3400 \\
\hline & 2400 & & 0.038 & 0.011 & 0.015 & 0 & 0 \\
\hline & 2600 & 0.486 & & 0.012 & 0.045 & 0 & 0 \\
\hline & 2800 & 0.675 & 0.675 & & 0.048 & 0.004 & 0.001 \\
\hline & 3000 & 0.767 & 0.675 & 0.525 & & 0.016 & 0.004 \\
\hline & 3200 & 1 & 1 & 0.916 & 0.888 & & 0.004 \\
\hline & 3400 & 1 & 1 & 0.971 & 0.971 & 0.685 & \\
\hline
\end{tabular}

Los valores cercanos a 1 corresponden al mayor recambio o anidamiento.

Values close to 1 correspond to the greater turnover or nestedness.

El análisis de disimilitud en la composición de especies en las diferentes altitudes arrojó claramente tres agrupaciones que corresponden a las zonas de vida, zona paramuna (3200 - $3400 \mathrm{~m})$, bosque alto andino (2800 - $3000 \mathrm{~m})$ y bosque andino (2400 - $2600 \mathrm{~m})$; la similitud entre cada una de las diferentes altitudes fue menor al $50 \%$ de la composición de especies. Por su parte el análisis de diversidad beta según Baselga (2010) arrojó como resultado una diversidad beta total de 0.864 para el gradiente altitudinal; este valor fue alto debido a el recambio de especies entre las altitudes $(\beta \mathrm{SIM}=0.852)$, el anidamiento o diferencias en la riqueza aportó un valor muy bajo ( $\beta \mathrm{SNE}$ $=0.012$ ). Adicionalmente se utilizó el concepto de Bratio propuesto por Dobrovolski, Melo, Cassemiro y Diniz-Filho (2012) y Si, Baselga y Ding (2015), como la relación $\beta$ SNE/ $\beta S O R=$ 0.013 ; el resultado de $\beta$ ratio $<0.5$ permite concluir que los procesos de diversidad beta están siendo provocados por el recambio de especies a lo largo del intervalo altitudinal (Cuadro 2).

En cuanto a la distribución de especies a través del intervalo altitudinal, ninguna de las especies se distribuyó en todas las altitudes, aunque Lepidozia cupressina, Lophocolea muricata, A. conferta, Metzgeria albinea y Microlejeunea bullata se encontraron en toda la zona de bosque (2400-3000 m), mientras que Drepanolejeunea sp2 se ubicó desde el bosque alto andino hasta la región paramuna $(2800-3400 \mathrm{~m})$. La zona altitudinal con el mayor número de especies únicas se ubicó en los $3400 \mathrm{~m}$ con 24 especies, seguida de 3000 y $3200 \mathrm{~m}$ con 22 especies cada una (Fig. 2). Sólo dos especies únicas se ubicaron de los 2400 hasta los $2800 \mathrm{~m}$; Calypogeia peruviana y Lejeunea flava, mientras que entre los 2600 y $3000 \mathrm{~m}$ se encontraron tres especies únicas Metzgeria ciliata, Lejeunea sp5 y Lejeunea sp11, mientras que Bazzania stolonifera y Telaranea nematodes se ubicaron desde los 2800 hasta los $3200 \mathrm{~m}$.

Los factores ambientales mostraron diferencias significativas en cada una de las altitudes evaluadas (ANOVA $\mathrm{P}<0.05$ ), además la luminosidad aumenta mientras que la temperatura desciende altitudinalmente. El análisis de correspondencia Canónico (CCA) evidenció una distribución de especies y puntos de muestreo dados por los vectores de las variables ambientales agrupándose por su zona de vida, aunque se observó una división marcada entre la zona de bosque y de páramo. Los dos primeros ejes explican el 27 y $18 \%$ de la varianza total sobre la distribución de especies, además estos dos ejes fueron significativos (Monte Carlo $\mathrm{P}$ $<0.01$ ), por ello se utilizaron para visualizar los datos. El CCA mostró que los factores que 


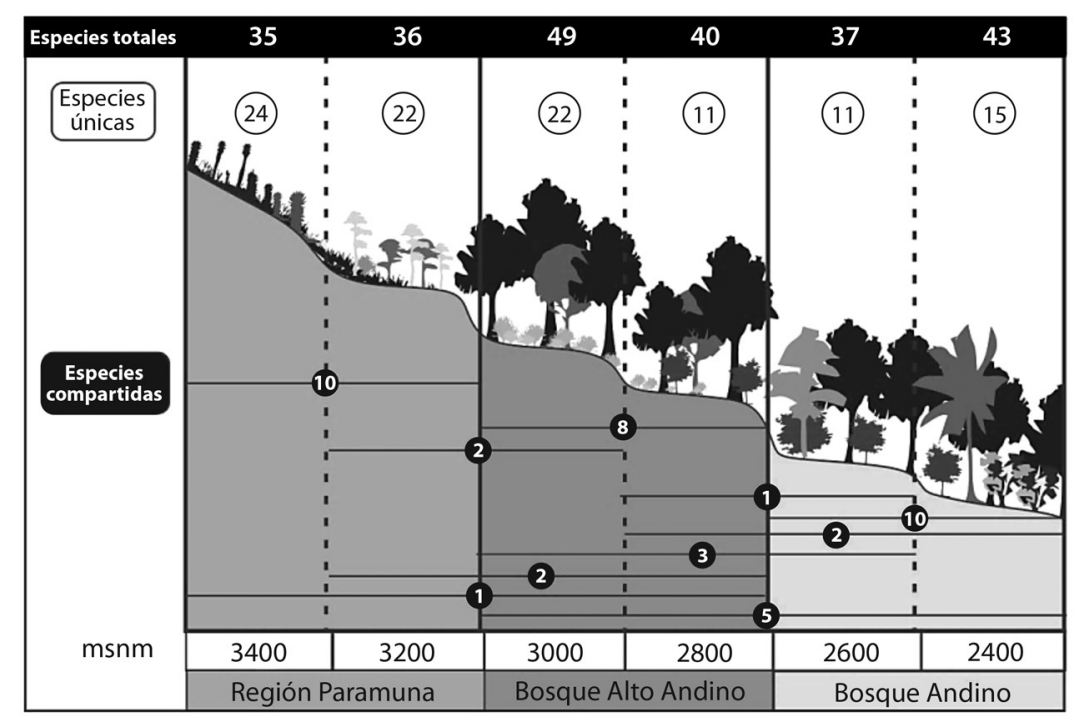

Fig. 2. Distribución altitudinal de las hepáticas a través del intervalo altitudinal y en las diferentes zonas de vida. Especies totales (barra superior negra), especies únicas (círculos superiores grises) y especies compartidas (círculos inferiores negros) en diferentes altitudes.

Fig. 2. Altitudinal distribution of the liverworts throughout the altitudinal interval and in the different zones of life. Total species (upper black bar), unique species (gray upper circles) and shared species (black lower circles) at different elevations.

más influyen en la distribución altitudinal de las hepáticas son la luminosidad (L y Ls) y la temperatura (Tmin y Tmax) (Fig. 3).

\section{DISCUSIÓN}

Se obtuvo una diversidad considerablemente alta en una pequeña franja altitudinal de tan solo $1000 \mathrm{~m}$, registrándose un $22 \%$ de las especies de hepáticas presentes en el territorio colombiano (Campos et al., 2014). Se esperaba una gran riqueza de especies en esta zona ya que como explican Uribe y Gradstein (1999), en la región Andina se encuentra cerca del 90\% de las especies registradas para Colombia. La gran diversidad de especies en esta región se debe a las condiciones ambientales de luminosidad, temperatura y humedad constantes que favorecen el desarrollo de las hepáticas, así como otros grupos de plantas epifitas, provocados por el levantamiento de los Andes del norte (Wolf 1993, 1994).

El hábito folioso mostró una clara dominancia sobre el taloso, siendo predominante en las hepáticas encontradas en el intervalo altitudinal con casi el $90 \%$ de las especies presentes en la zona. Las hepáticas foliosas son más resistentes a los cambios en la humedad del ambiente y pueden mantener un estado de latencia por más tiempo mientras que las especies talosas se encuentran restringidas a sitios con fuentes hídricas cercanas o con una humedad muy elevada y con luminosidad muy baja (Lagos et al., 2008). Los resultados concuerdan con los trabajos de Álvaro, Díaz y Morales (2007), Gil y Morales (2014), Ruiz y Aguirre (2003) y Vargas y Morales (2014) realizados en los Andes colombianos en los que el hábito de crecimiento dominante fue la forma foliosa. Por ello la familia con mayor riqueza y abundancia fue Lejeuneaceae, ya que las especies de esta familia se distribuyeron por todo el gradiente; esto era de esperar, ya que Gradstein (1994) indicó que ésta es una de las familias con mayor riqueza de especies en Colombia. A pesar de ello Plagiochila tuvo la mayor riqueza de especies (31 especies) y con la mayor cobertura por género con 9000 


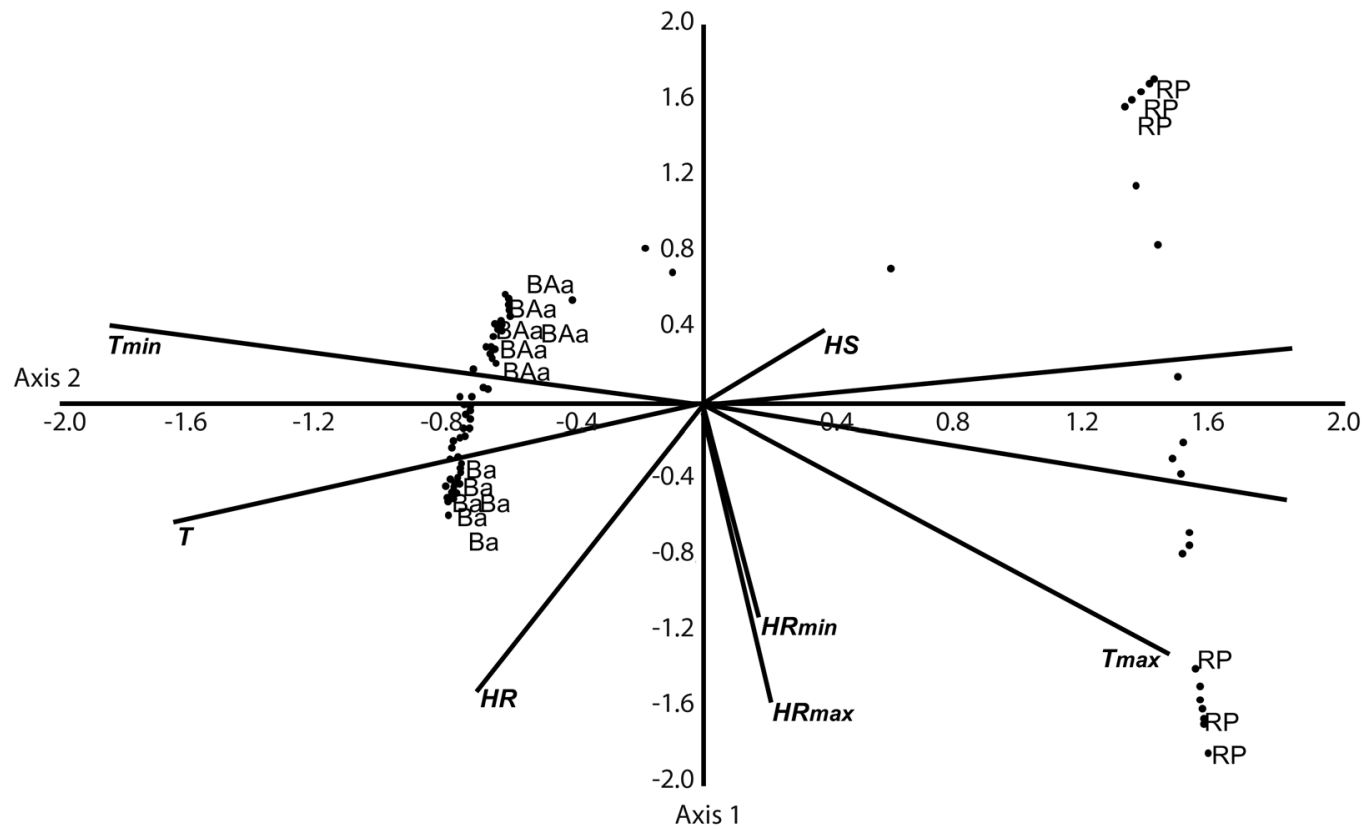

Fig. 3. Análisis de correspondencia canónica relacionando las variables ambientales y el índice de valor de importancia de cada especie. Ba: Bosque andino, BAa: Bosque alto andino, RP: Región paramuna, Hs: Humedad suelo, Ls: Luminosidad suelo, L: Luminosidad, T: Temperatura, HR: Humedad relativa, Tmax: Temperatura máxima, Tmin: Temperatura mínima, HRmax: Humedad relativa máxima, HRmin: Humedad relativa mínima.

Fig. 3. Canonical correspondence analysis relating the environmental variables and the importance value index of each species. Ba: Andean forest, BAa: High Andean forest, RP: Region paramuna, Hs: Soil moisture, Ls: Soil brightness, L: Brightness, T: Temperature, HR: Relative humidity, Tmax: High temperature, Tmin: Low temperature, HRmax: High relative humidity, HRmin: Low relative humidity.

cm² $^{2}$, según Wolf (1993) es el género más rico en especies dentro del territorio colombiano, presentando un amplio rango de distribución y una cobertura mayor.

La zona paramuna específicamente a los $3400 \mathrm{~m}$ tuvo la mayor riqueza de familias y géneros de los registrados en todo el intervalo altitudinal, esto se debe en gran medida a la diferencia microclimática que presenta debido a la poca cobertura vegetal existente, afectando el establecimiento de las especies; la luminosidad es tres veces mayor que en la zona boscosa, así mismo se presentan cambios de temperatura muy bruscos, llegando a los $31{ }^{\circ} \mathrm{C}$ en el día y temperaturas bajo cero en las noches. Aparte de estos factores evaluados, el viento causa un gran efecto en el crecimiento de hepáticas ya que produce una mayor desecación y limita la reproducción en la misma zona. Los géneros y familias de hepáticas tienen especies únicas y poseen una baja riqueza taxonómica, esto podría ser resultado de las condiciones tan drásticas a las que se exponen las especies en este ambiente, por ello deben tener mecanismos biológicos para poder crecer en estas zonas tales como cutículas más gruesas, pigmentación rojiza y hojas imbricadas. Este resultado concuerda con el trabajo de Álvaro et al. (2007) realizado en el departamento de Boyacá a más de $3000 \mathrm{~m}$, donde se encontró un total de 77 especies distribuidas en 39 géneros y 22 familias.

De acuerdo con los resultados de riqueza de especies en el rango altitudinal evaluado, la diversidad de hepáticas mostró grandes diferencias entre los ensambles. El ensamble rico en especies a los $2800 \mathrm{~m}$ tuvo valores de diversidad más bajos que otros con menor riqueza, 
como a los $3200 \mathrm{~m}$, debido principalmente a la presencia de especies muy dominantes en los $2800 \mathrm{~m}$ y a un mejor reparto en la abundancia de especies en los $3200 \mathrm{~m}$. Aun así se presentó ensambles ricos en especies con altos valores de diversidad (Cuadro 1). La amplia diferencia entre la diversidad ${ }^{0} \mathrm{D}$ (riqueza) y el número de especies efectivas de las diversidades ${ }^{1} \mathrm{D}$ y ${ }^{2} \mathrm{D}$, indica que este ensamblaje presenta algunas especies abundantes y un gran número de especies raras, lo cual se ve reflejado en las abundancias relativas de cada especie. Los valores de diversidad ${ }^{1} \mathrm{D}$ y ${ }^{2} \mathrm{D}$ no reflejan un patrón de aumento o disminución relacionado a la altitud. Sin embargo, hay una mayor diversidad en los puntos de muestreo que corresponden al bosque andino y alto andino con una disminución de la diversidad en la región paramuna. Esto concuerda con el estudio de Uribe y Gradstein (1999) en el que se explica que la diversidad máxima de hepáticas en los Andes colombianos se encuentra entre los 2000 y 3000 m. Por encima de estas altitudes se disminuye la diversidad debido a las condiciones paramunas de alta luminosidad y baja humedad, limitando su crecimiento y dispersión. En ese mismo trabajo se encontró una relación directa entre la altitud y diversidad; sin embargo, esta afirmación es aplicable cuando se estudia un gradiente altitudinal mucho más amplio que el de este estudio. Según Rangel-Ch. (2015) la región subandina (1000 a $2000 \mathrm{~m})$ presenta la mayor diversidad de plantas con flores, musgos y hepáticas, mientras que la región paramuna registra los menores valores en diversidad de los grupos mencionados anteriormente. En comparación con otros grupos, Bolaños y Ramírez (2009) encontraron que hacia el sur en la cordillera Occidental colombiana la mayor diversidad de musgos se encuentra en la región subandina (1600 - $2100 \mathrm{~m})$.

Según los análisis de diversidad beta, la composición de hepáticas se encuentra más relacionada entre altitudes cercanas, ya que comparten condiciones microambientales similares. El análisis de ßratio evidenció una proporción $\beta \mathrm{SNE} / \beta \mathrm{SOR}<0.5$, lo que implica que la diversidad beta entre las diferentes alturas es ocasionada por el recambio de especies ( $\beta$ SIM), es decir el remplazo de especies de un lugar a otro (Baselga, 2010; Angeler, 2013; Si et al., 2015). La diversidad beta es alta entre las diferentes zonas de vida, correlacionándose con las altitudes, lo que quiere decir, que en cada zona de vida se presenta como mínimo 10 especies únicas, lo que provoca el recambio de especies en cada una de las altitudes de más del $30 \%$ y llegando en algunos casos hasta el $65 \%$ de cambio en la composición de especies hacia la región paramuna. Esta variación en la composición de especies en el gradiente altitudinal depende en gran medida del efecto de la estructura de la vegetación que influye en cada uno de los microambientes disponibles a las hepáticas (Ruiz \& Aguirre, 2003), además de los factores ambientales como temperatura y humedad propios de estas altitudes (Silva \& Porto, 2012; Silva et al., 2014). Según Santos y Costa (2010) el recambio de especies en los bosques de tierras bajas puede llegar a ser del $35 \%$ en un rango de $1000 \mathrm{~m}$, resultado cercano a los valores obtenidos en este estudio, que siendo mayores se relacionan con la gran diversidad de hepáticas en esta región.

La relación de la composición de especies con los factores ambientales de luminosidad, temperatura y humedad asociadas a las altitudes arrojó una alta dependencia con la temperatura y luminosidad. Estos factores disminuyen y aumentan respectivamente en el gradiente altitudinal y sufren variaciones significativas durante el año. La humedad por el contario es alta y se mantuvo relativamente constante en el tiempo por lo que no afecta en gran medida el establecimiento y crecimiento de las hepáticas. En el análisis del CCA se notó una clara diferenciación entre la región boscosa y paramuna, de la misma forma que el agrupamiento del análisis de diversidad beta; este hecho está relacionado más específicamente con la variación en luminosidad, factor que es muy importante en el establecimiento de las hepáticas, ya que cuando es muy alta limita su crecimiento por la cantidad de radiación que reciben, además de afectarlas en su regulación hídrica al causarles desecación. Por ello, las especies de la 
región paramuna deben ser más resistentes a este factor.

La temperatura afectó también la distribución de las hepáticas, separando las regiones boscosa y paramuna. En la región paramuna se presenta una muy alta variabilidad en este factor, altas temperaturas debido a la radiación solar diurna y bajas temperaturas en las noches hasta el punto de congelación. De manera contrastante, en la región boscosa la temperatura es más homogénea. La humedad relativa claramente divide la región boscosa en el bosque andino y bosque alto andino; el bosque alto andino presentó una mayor humedad a lo largo del año. La diversidad de hepáticas parece darse como respuesta a la interacción entre estos factores ambientales afectando así su distribución (Corrales et al., 2010; Delgado \& Ederra, 2013). Según Gignac (2001) las hepáticas son sensibles a eventuales cambios en la temperatura del planeta, cambiando su distribución y migrando hacia otras zonas. Para esta área geográfica, aquellas especies de distribución limitada en el gradiente altitudinal como Anastrophyllum auritum, serían más vulnerables a los cambios de temperatura y humedad ocasionados por fenómenos macroclimáticos.

De manera similar, en la distribución altitudinal de las hepáticas, la mayoría de las especies se encontraron restringidas a una única altitud. Este hecho las hace muy específicas de un microambiente que tiene una combinación particular de factores bióticos y abióticos, permitiendo establecer combinaciones de especies que se encuentran presentes en un hábitat determinado. Esta aparente especificidad las hace más vulnerables a cambios ambientales regionales. Según Santos y Costa (2010) las hepáticas pueden ser utilizadas como indicadores altitudinales y de zonas de vida, debido a sus relaciones con la vegetación vascular y los factores ambientales que afectan el recambio de especies en los diferentes ambientes de hasta un $30 \%$. Igualmente, Santos, Costa, Kinoshita y Shepherd (2014) plantean la idea de utilizar hepáticas como indicadores de zonas altitudinales ya que ejercen un filtro más preciso que la vegetación fanerógama para la determinación de ambientes o hábitats. Sin embargo especies como L. cupressina, L. muricata, A. conferta, M. albinea y $M$. bullata podrían ser menos vulnerables, ya que se encuentran en un rango de distribución altitudinal mayor.

En conclusión, a lo largo del gradiente altitudinal la composición de hepáticas varía ampliamente con un recambio de especies mayor al $30 \%$ cada $200 \mathrm{~m}$ altitudinales, además de albergar una gran variedad de especies únicas para cada altura. Se presenta una diversidad máxima en los $3000 \mathrm{~m}$ con casi el $30 \%$ de las especies encontradas en el gradiente, encontrando una alta relación entre la temperatura, humedad y luminosidad con la mayor riqueza de especies, además de registrarse diferentes especies de las familias Lejeuneaceae y Trichocoleaceae que pueden ser susceptibles a la humedad. De igual manera, la diversidad en las tres zonas de vida presentes varió, el bosque alto andino fue el que tuvo la mayor riqueza de especies, debido a condiciones de luminosidad y temperatura medias que determinan un microclima ideal para el establecimiento de la mayoría de las especies de hepáticas. A pesar de que la diversidad depende de la interacción de los factores ambientales evaluados, la luminosidad es el factor más importante en la distribución de las hepáticas en el intervalo altitudinal, limitando el crecimiento de diferentes especies de hepáticas y determinando otros factores críticos para su establecimiento como la temperatura y humedad. La cordillera Oriental representa para Colombia una zona muy diversa del territorio colombiano, llegado a encontrar un $22 \%$ de las especies colombianas en tan solo $1000 \mathrm{~m}$ altitudinales. La composición y distribución de especies de hepáticas en las diferentes altitudes demuestra la importancia de la conservación de los bosques andinos, ya que albergan una alta diversidad de hepáticas epífitas en los troncos y proporcionan materia orgánica para el establecimiento de otras especies de hepáticas.

\section{AGRADECIMIENTOS}

A la Universidad Industrial de Santander y al grupo de Estudios de Biodiversidad por 
el préstamo de sus instalaciones y financiamiento. A Jaime Uribe por sus consejos y sus bases en el estudio de hepáticas en Colombia y a la profesora María Eugenia Morales por su apoyo, consejos y por abrirnos las puertas del Herbario UPTC.

\section{RESUMEN}

La composición de especies de hepáticas sobre los Andes del Norte varía con la altitud en relación con diferentes factores bióticos (vegetación) y abióticos (temperatura, humedad y luminosidad). Para probar esta afirmación se determinó la diversidad y distribución de las Marchantiophytas en un rango altitudinal entre los 2400 y 3400 $\mathrm{m}$ sobre la Cordillera Oriental colombiana, estableciendo cómo éstas varían entre altitudes y zonas de vida con respecto a los factores abióticos. Se realizaron muestreos cada $200 \mathrm{~m}$ y se registraron datos ambientales durante un año. Se encontraron 162 especies, la composición de hepáticas varió a lo largo del gradiente altitudinal con un gran número de especies únicas en cada altitud. Se encontró la máxima diversidad en los $3000 \mathrm{~m}$, con una dominancia del hábito folioso y de especies epifitas, mientras que la mayor riqueza de familias y géneros se presentó entre los 3200 y $3400 \mathrm{~m}$. Anoplolejeunea conferta presentó la mayor cobertura en la zona así como el mayor valor del índice de valor de importancia (IVI) y distribución altitudinal más amplia, ubicándose desde los 2400 hasta $3000 \mathrm{~m}$ en cuatro diferentes sustratos. Las hepáticas se distribuyeron altitudinalmente de manera diferencial, con una diversidad beta alta (0.864) debido al recambio altitudinal de especies, con más del $30 \%$ de disimilaría en la composición de especies cada $200 \mathrm{~m}$ altitudinales, dependiendo principalmente de cambios en la luminosidad. Consecuentemente, la temperatura, humedad y luminosidad y su relación con la vegetación son determinantes en la diversidad y distribución de las hepáticas en la Cordillera Oriental de los Andes colombianos.

Palabras clave: ecología; briófitos; bosque andino; páramo; gradiente altitudinal.

\section{REFERENCIAS}

Aguirre, J. \& Ruiz, C. A. (2001). Composición florística de la brioflora de la Serranía del Perijá (CesarColombia): distribución y ecología. Caldasia, 23(1), 181-201.

Álvarez-Yépis, J. C., Martínez-Yrízar, A., Búrquez, A., \& Lindquist, C. (2008). Variation in vegetation structure and soil properties related tol and use history of old-growth and secondary tropical dry forest in northwestern Mexico. Forest Ecology and Management, 256, 355-366.
Álvaro, W., Díaz, M. P., \& Morales, M. E. (2007). Catálogo comentado de las hepáticas del Cerro de Mamapacha municipio de Chinavita-Boyacá Colombia. Acta Biológica Colombiana, 12(1), 67-86.

Angeler, D. G. (2013). Revealing a conservation challenge through partitioned long-term beta diversity: increasing turnover and decreasing nestedness of boreal lake metacommunities. Diversity and Distributions, 9, 772-81.

Barbosa, I., Uribe, J., \& Campos, L. (2007). Las hepáticas de Santa María (Boyacá Colombia) y alrededores. Caldasia, 29(1), 39-49.

Baselga, A. (2010). Partitioning the turnover and nestedness components of beta diversity. Global Ecology and Biogeography, 19, 134-143.

Baselga, A., \& Orme, C. D. L. (2012). Betapart: an R package for the study of beta diversity. Methods in Ecology and Evolution, 3, 808-812.

Bolaños, G. \& Ramírez, R. (2009). Distribución altitudinal de musgos en el municipio de Popayán, Cauca. Revista Asociación Colombiana de Ciencias Biológicas, $21,31-44$.

Campos, L., Gradstein, S. R., Uribe, J., \& ter Steege, H. (2014). Additions to the catalogue of Hepaticae of Colombia II. Cryptogamie Bryologie, 35(1), 77-92.

Carvalho, J. C., Cardoso, P., Borges, P. A. V., Schmera, D., \& Podani, J. (2013). Measuring fractions of beta diversity and their relationships to nestedness: a theoretical and empirical comparison of novel approaches. Oikos, 122, 825-834.

Carvalho, J. C., Cardoso, P., \& Gomes, P. (2012). Determining the relative roles of species replacement and species richness differences in generating beta-diversity patterns. Global Ecology and Biogeography, 21, 760-771.

Chao, A., Gotelli, N. J., Hsieh, T., Sander, E. L., Ma, K., Colwell, R. K., \& Ellison, A. M. (2014). Rarefaction and extrapolation with Hill numbers: a framework for sampling and estimation in species diversity studies. Ecological Monographs, 84, 45-67.

Chao, A., \& Jost, L. (2012). Coverage-based rarefaction and extrapolation: standardizing samples by completeness rather than size. Ecology, 93, 2533-2547.

Churchill, S., \& Linares, E., (1995). Prodromus bryologiae Novo-Granatensis. Introducción a la flora de musgos de Colombia. Parte 1 y parte 2 Colombia. Editora Guadalupe Ltda.

Corrales, A., Duque, A., Uribe, J., \& Londoño, V. (2010). Abundance and diversity patterns of terrestrial bryophyte species in secondary and planted montane forests in the northern portion of the Central cordillera of Colombia. The Bryologist, 113(1), 8-21. 
Crandall-Stotler, B., Stotler, R. E., \& Long, D. G. (2009). Phylogeny and classification of the Marchantiophyta. Edinburgh Journal of Botany, 66(1), 155-198.

Delgado, V., \& Ederra, A. (2013). Long-term changes (1982-2010) in the bryodiversity of Spanish beech forests assessed by means of Ellenberg indicator values of temperature nitrogen light and $\mathrm{pH}$. Biological Conservation, 157, 99-107.

Dobrovolski, R., Melo, A. S., Cassemiro, F., \& DinizFilho, J. (2012). Climatic history and dispersal ability explain the relative importance of turnover and nestedness components of beta diversity. Global Ecology and Biogeography, 21, 191-7.

Gelviz-Gelvez, S. M. \& Pavón-Hernández, N. P. (2013). Diversidad de especies arbustivas en una zona semiárida del centro de México. Revista Chapingo. Serie Ciencias Forestales y del Ambiente, 19, 323-335.

Gignac, L. (2001). Bryophytes as indicators of climate change. The Bryologist, 104(3), 410-420.

Gil, J. E. \& Morales, M. E. (2014). Estratificación vertical de briófitos epífitos encontrados en Quercus humboldtii (Fagaceae) de Boyacá Colombia. Revista de Biología Tropical, 62(2), 719-727.

Gradstein, S. R. (1994). Lejeuneaceae: Ptychantheae Brachiolejeuneae. Flora Neotropica, 62, 1-216.

Gradstein, S. R., Churchill, S., \& Salazar-Allen, N. (2001). Guide to the bryophytes of Tropical America. Memoirs of the New York Botanical Garden, $86,1-577$.

Gradstein, S. R., \& Costa, D. P. (2003). Liverworts and hornworts of Brazil. Memoirs of the New York Botanical Garden, 87, 1-317.

Hammer, O. D., Harper, A., \& Ryan, P. (2001). PAST: Paleontological statistics software package for education and data analysis. Paleontologia Electrónica, $4(1), 1-9$

Hilbe, J. (2007). STATISTICA 7: an overview. The American Statistician, 61, 91-94.

Hsieh, T. C., Ma, K. H., \& Chao, A. (2016). iNEXT: an R package for rarefaction and extrapolation of species diversity (Hill numbers). Methods in Ecology and Evolution, 7(12), 1451-1456.

Iwatsuki, Z. (1960). The epiphytic bryophyte communities in Japan. Journal Hattori Botanical Laboratory, 22, 159-339.

Jost, L. (2006). Entropy and diversity. Oikos, 113, 363-375.

Jost, L. (2010). The relation between evenness and diversity. Diversity, 2, 207-232.
Lagos, M. I., Sáenz, F. A., \& Morales, M. E. (2008). Briofitos retrofilos de tres quebradas del páramo de Mamapacha Chinavita (Boyacá-Colombia). Acta Biológica Colombiana, 13(1), 143-160.

Mota de Oliveira, S., \& ter Steege, H. (2013). Floristic overview of the epiphytic bryophytes of terra firme forests across the Amazon basin. Acta Botanica Brasilica, 27(2), 347-363.

Orrego, O. (2005). Briófitos de Caldas: La Reserva de Planalto. Boletín Cientifico-Centro de Museos - Museo de Historia Natural, 9, 31-50.

Pinzón, M. \& Linares, E. (2006). Diversidad de líquenes y briofitos en la región subxerofitica de la Herrera Mosquera (Cundinamarca-Colombia). I. Riqueza y estructura. Caldasia, 28(2), 243-257.

Rangel-Ch., O. (2000). Colombia diversidad biótica III: la región de vida paramuna de Colombia. Santafé de Bogotá DC. Universidad Nacional de Colombia.

Rangel-Ch., O. (2015). La biodiversidad de Colombia: significado y distribución regional. Revista de la Academia Colombiana de Ciencias Exactas, Físicas y Naturales, 39(51), 176-200.

Ruiz, C. A. \& Aguirre, J. (2003). Las comunidades de briófitos y su relación con la estructura de la vegetación fanerógama en el gradiente altitudinal de la Serranía del Perijá (Cesar-Colombia). Tropical Bryology, 24, 101-113.

Sánchez-González, A. \& López-Mata, L. (2003). Clasificación y ordenación de la vegetación del norte de la Sierra Nevada, a lo largo de un gradiente altitudinal. Anales del Instituto de Biología, Universidad Nacional Autónoma de México. Serie Botánica, 74, 47-71.

Santos, N. D., \& Costa, D. P. (2010). Altitudinal zonation of liverworts in the Atlantic Forest Southeastern Brazil. The Bryologist, 113(3), 631-645.

Santos, N. D., Costa, D. P., Kinoshita, L. S., \& Shepherd, G. J. (2014). Windborne: Can liverworts be used as indicators of altitudinal gradient in the Brazilian Atlantic Forest. Ecological indicators, 36, 431-440.

Si, X. Baselga, A., \& Ding, P. (2015). Revealing BetaDiversity patterns of breeding bird and lizard communities on inundated Land-Bridge Islands by separating the Turnover and Nestedness components. PLoS ONE, 10(5), e0127692.

Silva, J. B., Santos, N. D., \& Pôrto, K. C. (2014). Betadiversity: Effect of geographical distance and environmental gradients on the rocky outcrop bryophytes. Cryptogamie Bryologie, 35(2), 133-163.

Silva, M. P., \& Pôrto, K. C. (2012). Bryophyte communities along horizontal and vertical gradients in a human-modified Atlantic Forest remnant. Botany, 91(3), 155-166. 
Teeuwen, M. (1989). A revision of the genus Odontolejeunea (Spruce) Schiffn.(Leujeuneaceae Hepaticae). Nova Hedwigia, 48, 1-32.

Uribe, J. \& Aguirre, J. (1995). Las especies colombianas del género Symphyogyna (Hepaticae: Pallaviciniaceae). Caldasia, 17(82), 429-458.

Uribe, J., \& Aguirre, J. (1997). Clave para los géneros de hepáticas de Colombia. Caldasia, 19(1), 13-27.

Uribe, J. \& Gradstein, S. R. (1999). Estado del conocimiento de la flora de hepáticas de Colombia. Academia Colombiana Ciencias Exactas, Físicasmy Naturales, 23(87), 315-318.

Vargas, D. L. \& Morales, M. E. (2014). Hepáticas del Parque Natural Municipal "Robledales de Tipacoque" Boyacá-Colombia. Universitas Scientiarum, 19(3), 201-211.

von Konrat, M., Renner, M., Söderström, L., Hagborg, A., \& Mutke, J. (2008). Chapter nine: early land plants today: liverwort species diversity and the relationship with higher taxonomy and higher plants. Fieldiana Botany, 47, 91-104.

Wolf, J. H. (1993). Diversity patterns and biomass of epiphytic bryophytes and lichens along an altitudinal gradient in the northern Andes. Annals of the Missouri Botanical Garden, 80(4), 928-960.

Wolf, J. H. (1994). Factors controlling the distribution of vascular and non-vascular epiphytes in the northern Andes. Vegetatio, 112(1), 15-28.

Wolf, J. H. (1995). Non-vascular epiphyte diversity patterns in the canopy of an upper montane rain forest (2550-3670 m) Central Cordillera Colombia. Selbya$n a, 16(2), 185-195$.

Zepeda, C., Ávila, P., Díaz, U. S., Alanís, Y., Zarazúa, G., \& Amaya, A. (2014). Diversidad de musgos epifitos de la zona metropolitana del valle de Toluca México. Revista Mexicana de Biodiversidad, 85(1), 108-124. 